\title{
Introduction to the Special Section on Clinical High Risk for Mental Illness: Transdiagnostic Framework, Detection Strategies, Assessment, Treatment and Outcome
}

\author{
Tommaso Boldrini, ${ }^{1}$ Marco Solmi ${ }^{2,3}$
}

${ }^{1}$ Department of Developmental and Socialization Psychology, University of Padua, Padua; ${ }^{2}$ Neurosciences Department, University of Padua; Neuroscience Center, University Hospital of Padua, Psychiatry Unit, Padua, Italy; ${ }^{3}$ Early Psychosis: Interventions and Clinical Detection Lab, Department of Psychosis Studies, Institute of Psychiatry, Psychology \& Neuroscience, King's College London, London, UK

Historically, the idea of preventing severe mental disorders by intervening in their prodromal stages was first introduced by the psychoanalyst Harry Stack Sullivan. Sullivan (1994) claimed that schizophrenia was the result of maladaptive relationships and experiences, rather than hereditary or biological factors and, as such, could be prevented. As he wrote in the early 1900s: "I feel certain that many incipient [schizophrenic] cases might be arrested before the efficient contact with reality is completely suspended, and a long stay in institutions made necessary" (1927, pp. 106-107). The long journey which has led this original insight to the current framework of preventive psychiatry is intertwined with the development of the concept of schizophrenia, itself, and the major changes of its

Correspondence: Tommaso Boldrini, Department of Developmental and Socialization Psychology, University of Padova, via Venezia, 8, 35131, Padova, Italy.

Tel.: +39.0649917674.

E-mail: tommaso.boldrini@unipd.it

Citation: Boldrini, T., \& Solmi, M. (2020). Introduction to the Special Section on Clinical High Risk for Mental Illness: Transdiagnostic Framework, Detection Strategies, Assessment, Treatment and Outcome. Research in Psychotherapy: Psychopathology, Process and Outcome, 23(1), 1-3. doi: 10.4081/ripppo.2020.462

Key words: Psychosis; schizophrenia; psychosis risk; clinical high risk; early detection and prevention.

Conflict of interest: The authors declare no potential conflict of interest.

Received for publication: 23 April 2020.

Accepted for publication: 24 April 2020.

This work is licensed under a Creative Commons Attribution NonCommercial 4.0 License (CC BY-NC 4.0).

${ }^{\circ}$ Copyright: the Author(s), 2020

Licensee PAGEPress, Italy

Research in Psychotherapy:

Psychopathology, Process and Outcome 2020; 23:1-3

doi:10.4081/ripppo.2020.462 diagnostic formulation in the official diagnostic systems. In particular, the publication of DSM-III (American Psychiatric Association, 1980) in ' 80 have led to an "operational revolution" which radically transformed the psychiatric nosology and has remained almost entirely unchanged to this day. The diagnosis of schizophrenia was defined to strict diagnostic criteria which account only for the most severe and florid phenotypic expressions of schizophrenia spectrum disorders, without any room for subclinical psychotic symptoms. The paradigm of preventive psychiatry has recently stimulated renewed interest in milder psychotic clinical pictures that would have previously been considered "latent", "borderline", or "pseudoneurotic" schizophrenia in the pre-DSM-III era (Lingiardi \& Boldrini, 2019).

The last two decades of research led to substantial advances in knowledge and the most intensive implementation of preventive clinical strategies in the history of psychiatry. The 'Clinical High Risk' (CHR) criteria are a construct to identify young people at elevated risk for developing a psychotic disorder (Yung et al., 2008). Meeting the criteria for CHR is determined by either family history of schizophrenia or schizotypal personality disorder, or the presence of emergent or worsening attenuated positive symptoms, alongside a deterioration in social and occupational functioning. Longitudinal studies showed that CHR individuals typically have up to a 20 -fold increased in risk of developing psychosis compared to the general population (Fusar-Poli et al., 2016). Nonetheless, in recent years, it has become apparent that the some of the initial expectations were certainly inflated and that the overall ability of the current approach for altering the course of psychosis is still relatively modest (Fusar-Poli, 2018). Specifically, the risk of developing psychosis depends on the risk present before CHR criteria are met, and subjects with low pre-test risk (i.e. high life-time functioning, not referred by clinical context) keep a low risk of developing psychosis even if they score positive at interviews to defined CHR (Yung et al., 2007). Further concerns are related to the lack of a gold-standard intervention for CHR patients, as reveled by recent (network) meta-analyses 
(Modinos et al., 2014; Fusar-Poli et al., 2019), and to the inefficacy of current detection strategies which enable psychosis prevention services to identify only $5 \%$ of subjects who develop a schizophrenia spectrum disorders in the general population (Fusar-Poli, 2017). Moreover, CHR individuals present a large array of non-psychotic mental disorders (Fusar-Poli, Nelson, Valmaggia, Yung \& McGuire, 2014; Boldrini et al., 2019) which increasingly hinder the clinical management and treatment planning of such patients. Such limitations have created disillusionment and extreme polarities among researchers. On the one hand, some authors argue that there is sufficient evidence to completely discredit the HR paradigm (van Os \& Guloksuz, 2017); on the other hand, the founders of the HR paradigm claim that there is sufficient evidence to support it (McHugh et al., 2018; Yung et al., 2019).

This Special Section is composed by five contributions, highly multidisciplinary, which tap into this debate addressing critical issues related to early detection and intervention strategies for subjects at risk for psychosis.

In their study, Solmi, Campeol, and Cremonese (2020) described the clinical characteristics of subjects referring to a mental illness prevention services within an Italian mental health department which did not engage in any outreaching activities. Their rationale was based on previous evidence showing how outreaching activities decrease prognostic accuracy of at-risk mental state defining tools, over-attracting subjects who are not at increased risk of mental illness. Patients were evaluated with validated tools assessing functioning, at-risk mental state, schizotypal personality features, depressive and anxious symptoms, together with medical and family history collection. The authors showed how prevention service within mental health facility setting appears to properly detect subjects in need of treatment with a drop in functioning, at risk of developing severe mental illness.

Moving towards intervention strategies, Firth (2020) investigated the nature of physical activity and exercise as a pragmatic, tolerable and cost-effective treatment for CHR youths. In a greatly clear and well-structured review, the author reviewed existing literature for exercise as an adjunctive intervention for those treated for schizophrenia and other psychotic disorders, as well as preliminary evidence form studies on physical exercise as a standalone intervention in CHR individuals. Moreover the "lessons learnt" from exercise interventions in related populations (e.g., first-episode or established psychosis) were deeply discussed to inform strategies, considerations, and future research around implementing exercise as an early intervention for psychosis.

Pozza, Meneghelli, Melliante, Amato, and Déttore (2020) focused their investigation on the transdiagnostic construct of anxiety sensitivity (i.e., individual differences consisting of fear of arousal-relates sensations), which has been hypothesized to be a vulnerability and maintenance factor of the intensity/recurrence of attenuated psychotic symptoms in CHR individuals. Their results partially supported the cognitive model of psychosis-risk (van der Gaag, van den Berg, \& Ising, 2019), providing clinically useful insights regarding psychological treatments for CHR individuals routed in the cognitive behavioral approach.

By following a quite different theoretical orientation, the effort of Boldrini, Lo Buglio, Giovanardi, Lingiardi and Salcuni (2020) was to show how psychodynamic thinking can contribute to widen our knowledge in the vulnerability to severe mental disorders. In their article, the typical psychodynamic construct of defense mechanisms (i.e., psychological mechanisms that mediate the individual's reaction to emotional conflicts and to external stressors) has been assessed in CHR individuals via observer-rater methods applied to clinical interview transcripts. The clinical meaning of their investigation taps into the possibility to address the specific defense vulnerability of CHR patients within psychotherapeutic interventions, with the potential to restoring human intelligibility to the psychotic experience and promoting mentalizing abilities of patients.

Finally, the theoretical perspective proposed by Pontillo et al. (2020) is aimed to discuss the clinical significance and the prognostic value of UHR criteria when they have been applied in younger ages. Despite more than $25 \%$ of schizophrenia cases onset before the age of 18 (Arango, 2011), the application of the concept of CHR in children and adolescents is relatively recent and still represent an under-researched area. By reviewing recent literature in the field, the authors drown a specific clinical profile for CHR children and adolescents, discussing the lower rate of transition and the higher rate of suicidal thinking and behavior compared to adults. Such as an investigation reveal the urgent need to rethink preventive interventions also considering the age specificity of patients.

Thanking all the authors - and the reviewers - involved in this topic, our hope and expectations were to provide new insights on the state of the art of prevention in psychiatry, and possible future directions from service organization and treatment perspectives.

\section{References}

American Psychiatric Association. (1980). Diagnostic and statistical manual of mental disorders (3rd ed.). Washington, DC: Author.

Arango, C. (2011). Attenuated psychotic symptoms syndrome: How it may affect child and adolescent psychiatry. European Child and Adolescent Psychiatry. https://doi.org/10.1007/ s00787-010-0144-2.

Boldrini T., Lo Buglio G., Giovanardi G., Lingiardi V., Salcuni S. (2020). Specificities of Defense Mechanisms in Youths at High Risk of Developing Psychosis: An Empirical Investigation. Research In Psychotherapy: Psychopathology, Process and Outcome

Boldrini, T., Tanzilli, A., Pontillo, M., Chirumbolo, A., Vicari, S., \& Lingiardi, V. (2019). Comorbid personality disorders 
in individuals with an at-risk mental state for psychosis: a meta-analytic review. Frontiers in Psychiatry. https://doi.org/ 10.3389 /fpsyt.2019.00429

Firth, J. (2020). Using Exercise to Improve Outcomes in those at-risk for Psychosis. Research In Psychotherapy: Psychopathology, Process and Outcome

Fusar-Poli, P. (2017). Extending the benefits of indicated prevention to improve outcomes of first-episode psychosis. JAMA Psychiatry. https://doi.org/10.1001/jamapsychiatry. 2017.1009

Fusar-Poli, P. (2018). The hype cycle of the clinical high-risk state for psychosis: The need of a refined approach. Schizophrenia Bulletin, 44(2), 250-253. https://doi.org/10.1093/ schbul/sbx 181

Fusar-Poli, P., Cappucciati, M., Borgwardt, S., Woods, S. W., Addington, J., Nelson, B., (...) McGuire, P. K. (2016). Heterogeneity of psychosis risk within individuals at clinical high risk: A meta-analytical stratification. JAMA Psychiatry. https://doi.org/10.1001/jamapsychiatry.2015.2324

Fusar-Poli, P., Davies, C., Solmi, M., Brondino, N., De Micheli, A., Kotlicka-Antczak, M., (...) Radua, J. (2019). Preventive Treatments for Psychosis: Umbrella Review (Just the Evidence). Frontiers in Psychiatry, 10(December). https://doi. org/10.3389/fpsyt.2019.00764

Fusar-Poli, P., Nelson, B., Valmaggia, L., Yung, A. R., \& McGuire, P. K. (2014). Comorbid depressive and anxiety disorders in 509 individuals with an at-risk mental state: impact on psychopathology and transition to psychosis. Schizophrenia Bulletin, 40(1), 120-131. https://doi.org/10.1093/ schbul/sbs 136

Lingiardi, V., \& Boldrini, T. (2019). The Diagnostic Dilemma of Psychosis. The Journal of Nervous and Mental Disease. https://doi.org/10.1097/nmd.0000000000001008

McHugh, M. J., McGorry, P. D., Yuen, H. P., Hickie, I. B., Thompson, A., de Haan, L., (...) Nelson, B. (2018). The Ultra-High-Risk for psychosis groups: Evidence to maintain the status quo. Schizophrenia Research. https://doi.org/ 10.1016/j.schres.2017.09.003

Modinos, G., Allen, P., Frascarelli, M., Tognin, S., Valmaggia, L., Xenaki, L., (...) Fusar-Poli, P. (2014). Are we really map- ping psychosis risk? Neuroanatomical signature of affective disorders in subjects at ultra-high risk. Psychological Medicine. https://doi.org/10.1017/S0033291714000865.

Pontillo M., Averna R., Tata M.C., Gargiullo P., Guerrera S., Vicari S. (2020). Clinical profile, conversion rate and suicidal thinking and behavior in children and adolescents at Ultra-High-Risk for psychosis: A Theoretical Perspective. Research in Psychotherapy: Psychopathology, Process and Outcome. In press.

Pozza A., Meneghelli A., Meliante M., Amato L., \& Dèttore D. (2020) Anxiety sensitivity dimensions in at-risk-mental states. Research In Psychotherapy: Psychopathology, Process and Outcome

Solmi M., Campeol M., Cremonese C. (2020). Clinically appropriate detection rates of a mental illness prevention service. Research In Psychotherapy: Psychopathology, Process and Outcome. In press.

van der Gaag, M., van den Berg, D., \& Ising, H. (2019). CBT in the prevention of psychosis and other severe mental disorders in patients with an at-risk mental state: A review and proposed next steps. Schizophrenia Research. https://doi.org/10.1016/j.schres.2017.08.018.

van Os, J., \& Guloksuz, S. (2017). A critique of the "ultra-high risk" and "transition" paradigm. World Psychiatry. https://doi.org/10.1002/wps.20423.

Yung, A. R., Nelson, B., Stanford, C., Simmons, M. B., Cosgrave, E. M., Killackey, E., ... McGorry, P. D. (2008). Validation of "prodromal" criteria to detect individuals at ultra-high risk of psychosis: 2-year follow-up. Schizophrenia Research. https://doi.org/10.1016/j.schres.2008.07.012.

Yung, A. R., Wood, S. J., Malla, A., Nelson, B., McGorry, P., \& Shah, J. (2019). The reality of at-risk mental state services: a response to recent criticisms. Psychological Medicine. https://doi.org/10.1017/s003329171900299x.

Yung, A. R., Yuen, H. P., Berger, G., Francey, S., Hung, T. C., Nelson, B., ... McGorry, P. (2007). Declining transition rate in ultra-high risk (prodromal) services: Dilution or reduction of risk? Schizophrenia Bulletin. https://doi.org/10.1093/ schbul/sbm015. 